\title{
Exit, Sunk Costs and the Selection of Firms
}

\author{
Yves Richelle \\ University of Laval (Quebec) \\ and \\ Paolo G. Garella \\ Universitá di Bologna
}

march 1995

JEL Classification : L13, L41

\section{Abstract}

The paper analyzes the question of which cost characteristics are exhibited by the firms that exit an oligopolistic market when costs are asymmetric and firms can credibly be forced out by the remaining competitors. The main results are: (i) if reentry is impossible (due to the presence of large sunk costs), then the firm with the highest marginal cost function dtays in; if reentry is costless then the firm with the highest average cost exits. Consequenty sunk costs not only affect the number of firms in an industry, but they also enter the determination of the type of firms that resist predation.

Keywords: endogenous coalition formation, exit, sunk costs. 


\section{Introduction}

In markets where firms differ as to their cost functions is it possible to predict what are the cost characteristics of the firms that stay or that exit? In perfectly competitive markets one can predict that the firms exiting the market are those with highest average costs. This prediction has been extended by Ghemawat and Nalebuff (1985), (1990) and Fudenberg and Tirole (1986) to the case of declining industries with few competitors. Their analyses show indeed that, in a war of attrition, the less efficient firm will be the first to exit $^{1}$. However many recent works (see chapters 8 and 9 in Tirole (1988) and Wilson (1992)) show that exit can occur in a wide variety of circumstances. We are therefore led to ask if the above prediction continue to hold in imperfectly competitive markets where firms are not engaged in a war of attrition.

This paper argues that the exiting firm may be the one with the lowest average cost function. To identify the basic argument leading to this conclusion, consider the following example. Three firms decide, at a first stage, to stay in the market or to exit and, at a second stage, those that stay decide how much to produce. All firms have identical fixed costs. They also have constant marginal costs with firm $i$ 's marginal cost being strictly smaller than firm $j$ 's marginal cost which, in turn, is strictly smaller than firm $k$ 's. Firms can therefore be ranked according to their average cost function with firm $i$ having the lowest one.

Then suppose that if all firms stay in the market, each of them will obtain a strictly negative profit at the Cournot equilibrium while, if only two firms stay in, their Cournot profit is positive and the third firm receives a zero profit. It immediately follows that for each couple of firms to stay in the market and to produce their Cournot quatity is an equilibrium of the two stage game. They are therefore three equilibria and a prediction on the cost characteristics of the exiting firm cannot be based only on this two stage game. Note, incidentally, that these equilibria are all Pareto efficient so that one cannot use coalitional proofness (see Bernheim, Peleg and Whinston

\footnotetext{
${ }^{1}$ For instance, Ghemawat and Nalebuf (1985) show that in a war of attrition with complete information where firms differ according to their production capacity, the biggest firm is the first to exit. But these authors assume that firms incur only a flow maintenance cost which is proportional to their capacity. Accordingly, the biggest firm is the one with the highest average cost function.
} 
(1987)) to select one or the other.

A possible route to follow for obtaining a prediction is indicated by the literature on endogenous coalition formation, as in Aumann and Myerson(1988), Gul (1989), and especially Bloch (1990a) and (1990b). These works use a non-cooperative sequential game to analyze the formation of coalition structures. In the same way, one can assume that a coalition formation game precedes the play of a game of the kind illustrated by the example above. We here adopt a specification of the coalition formation game where each firm in turn makes a declaration consisting of (i) a set of firms that stay in, (ii) a payoff vector for the three firms that can be obtained by the play of a non-cooperative equilibrium of the two stage game. One can interpret these declarations as "offers", and we model the acceptance (refusal) of an offer as the making of an identical (different) declaration. Since a declaration corresponds to one equilibrium, if two firms make the same declaration they agree to play the same equilibrium. This determines which equilibrium is played and the payoffs to all firms irrespective of the declaration made by the third firm.

It is important to realize that once two firms have adopted their equilibrium strategies the third firm has no better alternative than the play of its own best reply to those strategies, which coincides with the strategy specified in the equilibrium chosen by the other two firms. Therefore the way payoffs are determined has nothing to do with the application of a majority rule in collective decision making.

For further reference we call this sequential game "the cartel formation game". The equilibrium of this game gives a prediction of the firm that exits. For each order in which firms declare, there will be a unique subgame perfect equilibrium outcome in the cartel formation game. But, as one can expect, the equilibrium outcome will in general depend on the order of declaration. We are nevertheless able to show that, as long as a firm exits the market at the equilibrium, the cost characteristics of this firm can be identified and are independent of the order of declaration. The cartel formation game will therefore provide a strong prediction on the characteristics of the exiting firm.

In the example the unique equilibrium is with firms $j$ and $k$ making the same declaration of the form (i) $\{j, k\}$, and (ii) payoff zero for firm $i$, and Cournot payoffs for $j$ and $k$. A proof of this statement is trivial. Indeed the Cournot profit of a firm is increasing in the marginal cost of its rival which 
implies that firm $j$ makes the highest equilibrium profit when $k$ stays on the market and firm $k$ makes the highest equilibrium profit when $j$ stays on. Hence the firm exiting the market is the one with the lowest average costs, and not with the highest, as it would be predicted in a war of attrition or in perfect competition.

One is led to wonder if the difference in prediction could disappear if firms play a supergame instead of a one-shot game. Indeed, in a supergame, firms are generally able to maximize joint profits and, since joint profit maximization requires the minimization of variable cost, they will be induced to internalize the gain made by having an efficient partner.

In what follows we shall generalize the example given above by considering a general cost function and a production game consisting of an infinite repetition of the two-stage game of the example. In this game, we say that a two-firm cartel is feasible if there exists an equilibrium of the production game where these firms stay in and the third stays out along the equilibrium path. Obviously the analysis is interesting only if the production game displays at least two different feasible cartels.

The main results are that (i) if reentry is impossible, then the firm with the highest marginal cost function stays on at all equilibria of the cartel formation game, for any order of declaration, while fixed costs only determine the set of feasible cartels. (ii) If reentry is possible then the firm with the highest average cost exits. These results imply that the difference in prediction does not depend on the possibility or not to collude, but rather depends upon the existence or not of sunk costs for reentry.

A novel implication of the presence of sunk costs appears here: not only, as it is already well known from the literature on entry preemption, they can determine the number of firms, but they also enter the determination of the type of firms that stay in a market.

The paper is organised as follows: in the next Section we introduce our assumptions relative to the cost and demand functions and we analyse the equilibrium outcomes of the production game. In Section 3, the cartel formation game is formally presented. Our results are stated in Section 4 for the unprofitable reentry case and in Section 5 for the case of costless reentry. In Section 6 we test the robustness of the results for the case of unprofitable reentry to changes in the cartel formation game. The results stated in section 4 are shown to go through. Section 7 presents some concluding considerations to relate the results to the literature on transaction cost economics. 


\section{The production game}

We consider a supergame involving three firms. We shall denote this game by $\Gamma_{\alpha}$ and the set of firms by $N . \Gamma_{\alpha}$ consists of the infinite repetition of the two-stage game where (i) at the first stage each firm decides to stay in or to stay out of the market and (ii) at the second stage the firms which have decided to stay in the market, hereafter referred to as the active firms, play an usual Cournot game whilst an inactive firm produces nothing. At each stage decisions are made simultaneously and actions taken at the first stage are perfectly observed by all firms before they choose their production at the second stage. The scalar $\alpha$, belonging to the open interval $(0,1)$, denotes the discount factor common to all firms.

The purpose of this preparatory section is twofold. On the one hand, we give a precise content to the concept of a feasible cartel. On the other hand, for each feasible cartel $s$, we characterize the set of all payoff vectors that firms can obtain at a subgame perfect Nash equilibrium of $\Gamma_{\alpha}$ where along the equilibrium path only the firms in cartel $s$ stay in the market at each period.

To simplify the exposition we shall proceed in three steps. First, in subsection 2.1., we shall introduce the assumptions on the cost and demand functions. In the second step, in subsection 2.2., we shall ignore the first stage of the constituent game and concentrate on the game $\Gamma_{\alpha}(s)$ consisting of the infinite repetition of the Cournot game where the set of players is given by $s$ (i.e. firms in $s$ decide to stay in the market at every period and the firm outside $s$, if $s \neq N$, decides to stay out of the market at every period). We can thereby use the results from the literature on infinitely repeated games to bring forth a characterization of the set of equilibrium payoff vectors of $\Gamma_{\alpha}(s)$. In the last step, subsection 2.3., we introduce the possibility for each firm to exit the market. This will allow us to define what we mean by a feasible cartel and characterize, for each feasible cartel, the set of attainable payoff vectors $\mathcal{V}_{\alpha}(s)$.

\subsection{Assumptions}

An active firm has to pay a (time-invariant) fixed cost $F_{i}$ as well as a variable cost given by the function $c\left(q_{i} ; \theta_{i}, \bar{q}_{i}\right)$ where $\theta_{i}$ and $\bar{q}_{i}$ are (time-invariant) firmspecific parameters and $q_{i}$ stands for quantity (the time index that should be 
assigned to the quantity variable is omitted as long as this does not create confusion). If a firm decides to stay out, it produces nothing and incurs no cost. Furthermore if a firm, say $i$, has decided to stay out at period $t-1$, it must pay a reentry cost, $R_{i}$, if it decides to stay in the market at period $t$. We simplify the analysis by considering in turn two polar cases namely:

Assumption 1 Reentry is unprofitable i.e. $R_{i}$ is as large as we want, for $i=1,2,3$.

Assumption 2 Reentry is costless i.e. $R_{i}=0$, for $i=1,2,3$.

The variable cost function $c$ depends upon two firm-specific parameters, $\theta_{i}$ and $\bar{q}_{i} \cdot \bar{q}_{i}$ stands for the firm $i$ 's capacity constraint which means that, for a given $\theta_{i}$, firm $i$ cannot produce more than $\bar{q}_{i}$ and accordingly $c$ is only defined for $0 \leq q_{i} \leq \bar{q}_{i}$. On the other hand, $\theta_{i}$ is a convenient way to rank firms according to their marginal cost function. We shall indeed suppose that for any quantity $q$ such that the marginal cost to produce this quantity is well defined for firms $i$ and $j$, firm $j$ 's marginal cost is strictly greater than the one of firm $i$ if and only if $\theta_{j}>\theta_{i}$. More precisely way, let $X_{i}=\left[0, \bar{q}_{i}\right]$, then our assumptions regarding the variable cost function of any firm are the following:

Assumption 3 Let $\bar{q}_{i}>0$. The variable cost function is twice continuously differentiable with respect to $q_{i}$ and $\theta_{i}$ on $X_{i} \times R_{++}$. In addition, $c$ satisfies the following properties:

$$
\begin{array}{ll}
\text { 1. } & c\left(0 ; \theta_{i}, \bar{q}_{i}\right)=0, \forall \theta_{i} \in R_{++}, \\
\text {2. } \left.0 \leq \partial c\left(q_{i} ; \theta_{i}, \bar{q}_{i}\right) / \partial q_{i} \leq \eta_{i}, \eta_{i} \in\right] 0, \infty\left[\text {, and } \partial c\left(q_{i} ; \theta_{i}, \bar{q}_{i}\right) / \partial \theta_{i}>0, \forall\left(\theta_{i}, q_{i}\right) \in\right. \\
\quad R_{++} \times X_{i}, \\
\text { 3. } \partial^{2} c\left(q_{i} ; \theta, \bar{q}_{i}\right) / \partial q_{i} \partial \theta_{i}>0, \forall\left(\theta_{i}, q_{i}\right) \in R_{++} \times X_{i} .
\end{array}
$$

Now let $Q$ stand for aggregate output. At each period, the inverse demand function for the homogeneous good, denoted $f(Q)$, satisfies:

Assumption 4 For all $Q \in\left[0, \sum_{i=1}^{3} \bar{q}_{i}\right], f$ is twice continuously differentiable with $f(Q) \geq 0$ and $\partial f / \partial Q<0$. 
Note that we suppose that firms produce perfect substitutes in order to be able to concentrate ourselves only upon the influence of the cost characteristics on market structure.

For an active firm the profit function (gross of the reentry cost) in the Cournot game will be written as:

$$
\pi_{i}\left(q_{i}, Q_{-i}\right)=f\left(q_{i}+Q_{-i}\right) q_{i}-c\left(q_{i} ; \theta_{i}, \bar{q}_{i}\right)-F_{i}
$$

where $Q_{-i}=Q-q_{i}$. We shall assume:

Assumption $\mathbf{5}$ For all $i \in N, \pi_{i}$ is strictly quasi-concave on $X_{i} \times\left[0, \sum_{j \neq i} \bar{q}_{j}\right]$. Furthermore there exists $\left(q_{1}, q_{2}, q_{3}\right) \in X_{1} \times X_{2} \times X_{3}$ such that, for all $i$, $\pi_{i}\left(q_{i}, Q_{-i}\right)>0$.

Obviously these assumptions, together with the restriction that any active firm $i$ must choose a quantity in $\left[0, \bar{q}_{i}\right]$, are sufficient for the existence of a Cournot equilibrium. The second part of Assumption 5 will ensure, as we shall see later on, that there exists some "agreements" between the three firms with all of them remaining on the market. This could be assumed away, in fact simplifying the analysis without changing the results, but it is kept for the sake of generality.

The last restriction on the cost and demand functions is that whenever only two firms are active then, for any quantity its opponent can produce, a firm can achieve a positive profit. Formally, let us define $w_{i}(s)$ with $s=\{i, j\}$ as the minimal payoff firm $i$ can guarantee to itself when it faces firm $j$, i.e.:

$$
w_{i}(i, j)=\min _{q_{j} \in X_{j}} \max _{q_{i} \in X_{i}} \pi_{i}\left(q_{i}, q_{j}\right)
$$

Under assumption $4 \pi_{i}$ is a strictly decreasing function of $q_{j}$. Therefore, defining $q_{i}^{R}\left(\bar{q}_{j}\right)=\arg \max _{q_{i} \in X_{i}} \pi_{i}\left(q_{i}, \bar{q}_{j}\right)$, we have:

$$
w_{i}(i, j)=\pi_{i}\left(q_{i}^{R}\left(\bar{q}_{j}\right), \bar{q}_{j}\right)
$$

We shall require:

Assumption 6 For all $i, j \in N, w_{i}(i, j) \geq 0$ and $q_{i}^{R}\left(\bar{q}_{j}\right)<\bar{q}_{i}$.

This will guarantee on the one hand that the market cannot be monopolized and on the other hand that there exist couples $\left(q_{i}, q_{j}\right)$ such that $\pi_{i}\left(q_{i}, q_{j}\right)>w_{i}(i, j)$. We now turn to the characterization of the set of subgame perfect equilibrium payoff vectors of the infinitely repeated game $\Gamma_{\alpha}(s)$ 


\subsection{Equilibrium payoffs in $\Gamma_{\alpha}(s)$}

The typical payoff for firm $i$ in the game $\Gamma_{\alpha}(s)$ is given by:

$$
P_{i}=(1-\alpha) \sum_{t=0}^{\infty} \alpha^{t} \pi_{i}\left(q_{i}, Q_{-i}\right)
$$

Infinitely repeated games with discounting have been extensively analysed in the literature. It has been established (see, for instance, Theorem 3.2 in Sorin (1992)) that the set of subgame perfect Nash equilibrium payoff vectors of $\Gamma_{\alpha}(s)$ converges (with respect to the Haussdorf topology) to the set of individually rational and feasible payoff vectors of the constituent game as the discount factor tends to one ${ }^{2}$. This is one of the version of the so-called Folk Theorem. Accordingly, for our purposes we only need to characterize the set of individually rational and feasible payoff vectors of the Cournot one-shot game where the set of players is given by $s$. This set is denoted $\mathcal{W}(s)$.

To begin with let us denote the set of feasible payoff vectors with three active firms by $\mathcal{F}(N)$ and the one with two active firms by $\mathcal{F}(i, j)$. Let $X=X_{i} \times X_{j} \times X_{k}, \mathcal{F}(N)$ and $\mathcal{F}(i, j)$ are given by:

$$
\begin{gathered}
\mathcal{F}(N)=\text { convex hull }\left\{\left(P_{1}, P_{2}, P_{3}\right) \mid \begin{array}{l}
\exists\left(q_{1}, q_{2}, q_{3}\right) \in X \text { such that } \\
\\
\left.\quad P_{i}=\pi_{i}\left(q_{i}, Q_{-i}\right) \text { for } i=1,2,3\right\} .
\end{array}\right. \\
\mathcal{F}(i, j)=\text { convex hull }\left\{\left(P_{i}, P_{j}\right) \mid \begin{array}{rl}
\exists\left(q_{i}, q_{j}, 0\right) \in X \text { such that } P_{i}=\pi_{i}\left(q_{i}, Q_{-i}\right) \\
\text { and } \left.P_{j}=\pi_{j}\left(q_{j}, Q_{-j}\right)\right\} .
\end{array}\right.
\end{gathered}
$$

When the three firms are active, each active firm can guarantee to itself a profit given by:

$$
w_{i}(N)=\min _{q_{j} \in X_{j}, q_{k} \in X_{k}} \max _{q_{i} \in X_{i}} \pi_{i}\left(q_{i}, q_{j}+q_{k}\right)
$$

Accordingly the set of individually rational and feasible payoff vectors when firms in $s$ being active and $\# s \geq 2$ is simply:

$$
\begin{array}{cl}
\mathcal{W}(s)=\left\{\left(P_{1}, P_{2}, P_{3}\right) \mid \quad\right. & \left(P_{i}\right)_{i \in s} \in \mathcal{F}(s), P_{k}=0 \text { for } k \notin s \\
& \text { and } \left.P_{i} \geq w_{i}(s) \forall i \in s\right\} .
\end{array}
$$

\footnotetext{
${ }^{2}$ Provided the set of individually rational and feasible points has a non-empty interior. This is clearly the case under Assumption 6.
} 
Then, denoting the set of perfect Nash equilibrium payoff vectors of $\Gamma_{\alpha}(s)$ by $\mathcal{W}_{\alpha}(s)$, we have from the Folk Theorem that $\mathcal{W}_{\alpha}(s)$ converges to $\mathcal{W}(s)$ when $\alpha$ tends to one. We shall furthermore denote by $w_{\alpha i}(s)$ the minimal payoff firm $i$ can obtain in $\mathcal{W}_{\alpha}(s)$.

\subsection{Equilibrium payoffs in $\Gamma_{\alpha}$}

We now consider the game $\Gamma_{\alpha}$, namely the infinite repetition of the twostage constituent game where, at the first stage, firms decide to stay in or to stay out of the market and, at the second stage, active firms decide the quantity they produce. Let $\mathcal{V}_{\alpha}(s)$ denotes the set of attainable payoff vectors for cartel $s$. At the end of this section it will possible to characterize $\mathcal{V}_{\alpha}(s)$. Note that this set will depend on which of the two assumptions, 1 or 2 , is taken to hold.

To begin with, let us remark that, from assumption 5, there will always exist subgame perfect equilibria in $\Gamma_{\alpha}$ where along the equilibrium path the three firms stay in the market at each period. The set of payoff vectors which can be obtained at equilibria of this kind is denoted by $\mathcal{V}_{\alpha}(N)$ and it is easily verified that it coincides with $\mathcal{W}_{\alpha}(N) \cap R_{+}^{3}$. We shall furthermore denote by $e_{k}(N)$ a subgame perfect equilibrium of $\Gamma_{\alpha}$ where the three firms stay in at each period along the equilibrium path and where firm $k$ obtains a per-period profit equal to $\max \left\{0, w_{\alpha k}(N)\right\}$ and each other firm, say $h$, obtains a profit strictly greater than $\max \left\{0, w_{\alpha h}(N)\right\}^{3}$.

Then let us suppose that there is a firm, say $k$, such that $w_{\alpha k}(N)<0$. Consider first the existence of subgame perfect equilibria of $\Gamma_{\alpha}$ where firm $k$ stays out of the market at each period ${ }^{4}$. For $\alpha$ sufficiently close to one, the following strategy combination is a subgame perfect Nash equilibrium of the subgame starting after the three firms have decided to stay in the market at a given period: produce $\left(\bar{q}_{i}, \bar{q}_{j}, q_{k}^{R}\left(\bar{q}_{i}+\bar{q}_{j}\right)\right)$ followed by, wathever the quantity produced by firm $k$, either $e_{k}(N)$ if firms $i$ and $j$ have produced or

${ }^{3}$ Note that there are many output combinations that give firm $k$ a payoff equal to $w_{\alpha k}(N)$. For some of them the payoffs to $i$ and $j$ will be as specified in $e_{k}(N)$.

${ }^{4}$ We implicitly assume that firm $k$ 's outside opportunities has a per period value of zero. However the reader will easily verify that all our results go through if we have assumed that the per period value of firm $i$ 's outside opportunities, $i=1,2,3$, is given by a function $O\left(\theta_{i}\right)$ satisfying $\partial O / \partial \theta_{i} \geq 0$. The point is to define $\hat{w}_{\alpha i}(s)=w_{\alpha i}(s)-O\left(\theta_{i}\right)$ for all possible cartels and work with $\hat{w}_{\alpha i}(s)$ instead of $w_{\alpha i}(s)$. 
both did not produce $\bar{q}_{i}$ and $\bar{q}_{j}$ respectively, or $e_{i}(N)$ if firm $i$ did not produce $\bar{q}_{i}$ while firm $j$ has produced $\bar{q}_{j}$, or $e_{j}(N)$ if firm $j$ did not produce $\bar{q}_{j}$ while firm $i$ has produced $\bar{q}_{i}$. This is an equilibrium, in the subgame starting at the node where the three firms have entered the market, with firm $k$ having a negative payoff. Therefore using this equilibrium as a punishment triggered by the firm $k$ 's decision to stay in the market, firms $i$ and $j$ can force firm $k$ to stay out of the market at each period. We shall refer to this situation by saying that the cartel $\{i, j\}$ is feasible. It must be clear that the feasibility of a particular cartel, say $\{i, j\}$, neither excludes nor implies the feasibility of another cartel like $\{i, k\}$ for instance.

To sum up: we consider that the grand cartel, $N$, is always feasible since, by assumption 5, there always exist subgame perfect equilibria of $\Gamma_{\alpha}$ where, along the equilibrium path the three firms decide to stay in at each period. On the other hand we say that a two-firms cartel, say $\{i, j\}$, is feasible if and only if firms $i$ and $j$ can credibly predate firm $k$ i.e. $w_{\alpha k}(N)<0{ }^{5}$. We shall denote the set of feasible cartels by $\mathcal{S}$.

Before examining the set of equilibrium payoff vectors of a feasible cartel in the game $\Gamma_{\alpha}$, we must recognize that there is a third kind of market structure which could emerge namely the monopoly one. However this possibility is ruled out, whether reentry is costless or unprofitable, by assumption 6 .

We now turn to the characterization of the set of equilibrium payoff vectors that a feasible cartel, say $\{i, j\}$, can obtain in a subgame starting after the decision of firm $k$ to stay out of the market and the decisions of firms $i$ and $j$ to stay in the market. Consider first the case where the reentry cost is so high that it is never profitable for firm $k$ to decide to stay in the market at any period of this subgame (i.e. assumption 1 holds). In this situation assumption 6 will ensure that firms $i$ and $j$ will decide to stay in the market at each period of the subgame. This immediately implies that the set of equilibrium payoff vectors of the subgame coincides with the set of equilibrium payoff vectors of the game $\Gamma_{\alpha}(s)$, with $s=\{i, j\}$. Therefore we have $\mathcal{V}_{\alpha}(s)=\mathcal{W}_{\alpha}(s)$ for all feasible two-firms cartel $s$.

Consider now the case where reentry is costless, i.e. assumption 2 holds. Then the worst per-period payoff firm $i$ will obtain is equal to $\max \left\{0, w_{\alpha i}(N)\right\}$. Indeed consider the subgame which starts after firms $i$ and $j$ have decided

\footnotetext{
${ }^{5}$ The use of $w_{\alpha k}(N) \leq 0$ instead of $w_{\alpha k}(N)<0$ in the definition of a feasible cartel does not lead to any change in the analysis.
} 
to stay in while firm $k$ has decided to stay out of the market and either firm $i$ or firm $j$ has deviated from their respective quantity specified in a given strategy combination. Since reentry is costless, $e_{i}(N)$ and $e_{j}(N)$ are perfect equilibrium of this subgame. In other words, with costless reentry, firm $k$ can be used to punish a deviation by a member of the cartel so that the worst payoff a firm, say firm $i$, will obtain if it deviates from a specified quantity is simply the maximum between 0 and $w_{\alpha i}(N)$. Note that if cartel $\{j, k\}$ is feasible, if firm $i$ deviates it could be punished by its exclusion of the market leading to a zero payoff. But since cartel $\{j, k\}$ is feasible if and only if $w_{\alpha i}(N)<0$, the worst payoff firm $i$ will obtain is still equal to $\max \left\{0, w_{\alpha i}(N)\right\}$. It follows that the set of equilibrium payoff vector a feasible two-firm cartel $s$ can obtain under assumption $2, \mathcal{V}_{\alpha}(s)$, is simply equal to the following set:

$$
\begin{aligned}
\mathcal{V}_{\alpha}(s)=\left\{\left(P_{1}, P_{2}, P_{3}\right) \mid\right. & \left(P_{i}\right)_{i \in s} \in \mathcal{F}(s), P_{k}=0 \text { for } k \notin s, \\
& \text { and } \left.P_{i} \geq \max \left\{0, w_{\alpha i}(N)\right\} \forall i \in s\right\} .
\end{aligned}
$$

Note that, since $w_{\alpha i}(N)$ is strictly smaller than $w_{\alpha i}(s)$ for any $s \neq N$ to which $i$ belongs, then $\mathcal{V}_{\alpha}(s)$ is larger than $\mathcal{W}_{\alpha}(s)$.

\section{The cartel formation game}

Due to the multiplicity of equilibria in the production game, firms must coordinate upon the play of a particular equilibrium before the production game starts. The way firms solve this coordination problem is described by a three-stage game where at each stage one firm makes a declaration. The order in which declarations are made is exogeneously given and it is common knowledge.

A firm $i$ 's declaration, $d_{i}$, consists of a feasible cartel, $s_{i}$, to which firm $i$ must belong and of a payoff vector $p_{i}=\left(p_{i 1}, p_{i 2}, p_{i 3}\right)$ which must belong to $\mathcal{V}_{\alpha}\left(s_{i}\right)$. The set of firm $i$ 's declarations is thus $\mathcal{D}_{i}=\{(s, p) \mid i \in s, s \in$ $\mathcal{S}$ and $\left.p \in \mathcal{V}_{\alpha}(s)\right\}$. Furthermore let $\mathcal{H}_{i}$ denote the set of declarations preceding that of firm $i$ with $\mathcal{H}_{i}=\emptyset$ if firm $i$ is the first to make a declaration. Then a strategy for firm $i$ is a mapping $\sigma_{i}: \mathcal{H}_{i} \rightarrow \mathcal{D}_{i}$.

Let $d$ denote a declaration vector, firm $i$ will receive the payoff $g_{i}$ given 
by:

$$
g_{i}(d)=\left\{\begin{array}{lll}
p_{i i} & \text { iff } & \left\{\begin{array}{l}
\text { either } d_{i}=d_{j}, \\
\text { or } d_{i}=d_{k}, \\
\text { or } d_{i}=d_{j}=d_{k},
\end{array}\right. \\
p_{j i} & \text { iff } & d_{i} \neq d_{j} \text { and } d_{j}=d_{k}, \\
\bar{g}_{i} & & \text { otherwise. }
\end{array}\right.
$$

Accordingly firm $i$ will receive its proposed payoff $p_{i i}$ if its declaration is compatible with (identical to) either that of firm $j$ (i.e. $d_{i}=d_{j}$ ) or that of firm $k$ (i.e. $d_{i}=d_{k}$ ) or both. If the declarations of firms $j$ and $k$ are compatible then firm $i$ will obtain the payoff assigned to it in the firm $j$ 's declaration. This payoff assignment follows from the fact that, once two firms agree to play according to a partiacular equilibrium of the production game, the third one's best response in the production game is to play according to this equilibrium too. Consequently all firms will receive the payoff corresponding to the equilibrium upon which two firms have decided to coordinate. Finally, if all the declarations are pairwise incompatible no agreement is possible and firm $i$ will receive some predetermined payoff $\bar{g}_{i}$. We suppose that firm $i$ 's reservation payoff, $\bar{g}_{i}$, corresponds to the worst payoff it can obtain in the production game $\Gamma_{\alpha}$ or, in other words, the best payoff firm $i$ can guarantee to itself in this game. Accordingly ${ }^{6}, \bar{g}_{i}=\max \left\{0, w_{\alpha i}(N)\right\} \forall i \in N$. To end up note that since any strategy combination $\left(\sigma_{i}, \sigma_{j}, \sigma_{k}\right)$ leads to a declaration vector the payoff of firm $i$ in the cartel formation game will be given by $g_{i}$.

\section{Equilibria in the cartel formation game with unprofitable reentry}

We turn now to the characterization of the equilibria of the cartel formation game when, in the production game, reentry is unprofitable i.e. assumption 1 holds. To simplify the exposition we shall introduce some pieces of terminology: We shall say that, for a given order of declaration, say $(i, j, k)$, cartel $s$ forms if and only if, for this order of declaration, there exists a subgame perfect Nash equilibrium of the cartel formation game, $\sigma^{*}$, leading to a declaration vector $d^{*}$ defined by $\sigma_{i}^{*}=d_{i}^{*}, \sigma_{j}^{*}\left(d_{i}^{*}\right)=d_{j}^{*}, \sigma_{k}^{*}\left(d_{i}^{*}, d_{j}^{*}\right)=d_{k}^{*}$ such

\footnotetext{
${ }^{6}$ Recall that outside opportunities, if there exist, are taken into account in the stay in/stay out decision, see footnote 4.
} 
that:

$$
d_{h}^{*}=d_{l}^{*} \text { for } h, l \in s
$$

On the other hand, we shall say that cartel s will not form for a given order of declaration if and only if there does not exist at least one subgame perfect Nash equilibrium of the cartel formation game satisfying the above requirement.

As one could expect subgame perfect equilibria of the cartel formation game differ according to the order of declaration. However we can identify the cartels which do not form whatever the order of declaration. Let $W_{\alpha i}(s)$ be the highest one-period profit that firm $i$ can obtain in $\mathcal{W}_{\alpha}(s)$. Then,

Proposition 1 Suppose all our assumptions except assumption 2hold. Then there exists $\bar{\alpha}<1$ such that for all $\alpha \in(\bar{\alpha}, 1)$ and whatever the order of declaration we have:

1. A cartel forms,

2. if $\mathcal{S}=\{\{i, j\},\{i, k\}, N\}$ and $\theta_{j}<\theta_{k}$, then cartel $\{i, j\}$ does not form,

3. if $\mathcal{S}=\{\{i, j\},\{i, k\},\{j, k\}, N\}$ and $\theta_{i}<\theta_{j}<\theta_{k}$, then (a) cartel $\{i, j\}$ does not form and (b) if in addition $\bar{q}_{j} \leq \bar{q}_{i}$ and $W_{\alpha j}(i, j) \geq W_{\alpha j}(N)$ then cartels $\{i, j\}$ and $\{i, k\}$ do not form.

Fixed costs, marginal cost functions and capacity constraints determine all together which cartels are feasible i.e. which firms can be forced to stay out by the two others. However on the basis of marginal cost functions alone we can conclude that if the firm with the highest marginal cost function belongs to a feasible two-firms cartel then it will stay in the market. This means that a sufficient condition for the highest marginal cost firm to survive is the existence of a partner with which it can predate the third firm. Furthermore if in addition we suppose that $\bar{q}_{j} \leq \bar{q}_{i}{ }^{7}$ and $W_{\alpha j}(i, j) \geq W_{\alpha j}(N)$ then we can be more precise about the survival of the lowest marginal cost firm. Indeed in this case the results state that if a firm is forced to stay out of the market it

\footnotetext{
${ }^{7}$ As the reader will see in the proof of Lemma 4 the result will hold under the weaker but less transparent (sufficient) condition: $\pi_{i}\left(q_{j}^{R}\left(\overline{q_{i}}\right), \overline{q_{i}}\right) \leq \pi_{i}\left(q_{i}^{R}\left(\overline{q_{j}}\right), \overline{q_{j}}\right)$.
} 
is the one with the lowest marginal cost function once there exists a feasible cartel to which this firm does not belong. Accordingly a low marginal cost function and, since the results do not depend on the level of fixed costs, a low average cost function is not an advantage to survive in a market, if we except its role in the determination of the set of feasible cartels. Put in another way, our results suggest that to face predation the use of a technology leading to a small fixed cost and a (relatively) high marginal cost function do provide strong advantages with respect to the use of a technology leading to a large fixed cost and a low marginal cost function.

The cornerstone underlying our results is:

Lemma 1 Suppose all our assumptions except 2 hold and let $W_{\alpha i}(s)$ be the highest payoff firm $i$ can obtain in $\mathcal{V}_{\alpha}(s)$. Then there exists $\bar{\alpha}<1$ such that for all $\alpha \in(\bar{\alpha}, 1), W_{\alpha i}(i, j)<W_{\alpha i}(i, k)$ if and only if $\theta_{j}<\theta_{k}$.

Proof: Let $W_{i}(s)$ be the highest payoff firm $i$ can obtain in $\mathcal{W}(s)$. If we are able to prove that $W_{i}(i, j)<W_{i}(i, k)$ if and only if $\theta_{j}<\theta_{k}$ then the result will follow immediately by the application of the Folk Theorem.

It is obvious that:

$$
\begin{aligned}
& W_{i}(i, j)=\max _{q_{i} \in X_{i} q_{j} \in X_{j}} \pi_{i}\left(q_{i}, q_{j}\right) \text { subject to } \pi_{j}\left(q_{j}, q_{i}\right)=w_{j}(i, j), \\
& W_{i}(i, k)=\max _{q_{i} \in X_{i} q_{k} \in X_{k}} \pi_{i}\left(q_{i}, q_{k}\right) \text { subject to } \pi_{k}\left(q_{k}, q_{i}\right)=w_{k}(i, k) .
\end{aligned}
$$

Remark immediately that the constraints in these optimization programs do not depend on the fixed cost and hence the constraints differ only in the level of $\theta$.

For $q_{i}=\bar{q}_{i}$, the constraints in these maximization programs are satisfied if and only if $q_{j}=q_{j}^{R}\left(\bar{q}_{i}\right)$ and $q_{k}=q_{k}^{R}\left(\bar{q}_{i}\right)$ respectively. Under our assumptions we obviously have $q_{j}^{R}\left(\bar{q}_{i}\right)>q_{k}^{R}\left(\bar{q}_{i}\right)$ if and only if $\theta_{j}<\theta_{k}$. Futhermore for any given $q_{i} \in\left[0, \bar{q}_{i}\right)$ let us differentiate $\pi_{j}\left(q_{j}, q_{i}\right)=w_{j}(i, j)$ with respect to $q_{j}$ and $\theta_{j}$. We obtain:

$$
\frac{\partial \pi_{j}\left(q_{j}, q_{i}\right)}{\partial q_{j}} d q_{j}=\left[\frac{\partial c\left(q_{j} ; \theta_{j}, \bar{q}_{j}\right)}{\partial \theta_{j}}-\frac{\partial c\left(q^{R}\left(\bar{q}_{i}\right) ; \theta_{j}, \bar{q}_{j}\right)}{\partial \theta_{j}}\right] d \theta_{j} .
$$

where all derivatives are evaluated at $\left(q_{j}, q_{i}\right)$ such that $\pi_{j}\left(q_{j}, q_{i}\right)=w_{j}(i, j)$. Since $\pi_{j}$ is a strictly quasi-concave function we have that $\partial \pi_{j}\left(q_{j}, q_{i}\right) / \partial q_{j}$ is 
strictly negative for any $q_{j} \in\left(q^{R}\left(\bar{q}_{i}\right), \bar{q}_{j}\right]$ and strictly positive for any $q_{j} \in$ $\left[0, q^{R}\left(\bar{q}_{i}\right)\right)$. Moreover, by assumption 3, marginal cost is increasing in $\theta$ so that $\partial c\left(q_{j} ; \theta_{j}, \bar{q}_{j}\right) / \partial \theta_{j}-\partial c\left(q^{R}\left(\bar{q}_{i}\right) ; \theta_{j}, \bar{q}_{j}\right) / \partial \theta_{j}$ is strictly positive for any $q_{j} \in$ $\left(q^{R}\left(\bar{q}_{i}\right), \bar{q}_{j}\right]$ and strictly negative for any $q_{j} \in\left[0, q^{R}\left(\bar{q}_{i}\right)\right)$. It then follows that $d q_{j} / d \theta_{j}$ is strictly negative for any given $q_{i} \in\left[0, \bar{q}_{i}\right)$ and $q_{j}$ satisfying $\pi_{j}\left(q_{j}, q_{i}\right)=w_{j}(i, j)$. This establishes that the isoprofit curve $\pi_{j}\left(q_{j}, q_{i}\right)=$ $w_{j}(i, j)$ shifts rightwards in the coordinate $\left(q_{j}, q_{i}\right)$ as $\theta_{j}$ decreases. As shown in Figure 1 the result then follows.

As shown by the proof, the fixed cost does not matter since it affects both sides of the constraint in the maximization program in the same way. Hence, for any quantity produced by firm $i$, the quantity required to satisfy the constraint is independent of the fixed cost. On the other hand, the level of $\theta$, i.e. the level of the marginal cost for a given quantity produced, affects the constraint in two ways. First, if the right-hand side of the constraint were independent of $\theta$ then, in the coordinates of Figure 1 , firm $k$ 's isoprofit curve will be entirely below the firm $j$ 's one as long as $\theta_{j}<\theta_{k}$. This effect reflects the advantage to form a cartel with a low marginal cost firm. Second, however, the minimal payoff required by a firm to participate in a cartel with firm $i$ clearly decreases with $\theta$. This translates the intuition that a low marginal cost firm will be more greedy than a firm with a higher marginal cost. What the Lemma states is that the second effect dominates the first one.

According to this result, for $\theta_{k}$ larger than $\theta_{j}$, firm $k$ can always give to firm $i$ a greater payoff than the highest payoff firm $i$ can obtain with firm $j$. On the other hand if cartel $\{i, j\}$ forms then firm $k$ will receive a zero payoff while it will obtain at least $w_{\alpha k}(i, k)>0$ if cartel $\{i, k\}$ forms. Hence, loosely speaking, firm $k$ has always the opportunity and the willingness to prevent the formation of cartel $\{i, j\}$ so that this cartel cannot form.

Remark that we cannot exclude the formation of the grand cartel, $N$, for all orders of declaration. Indeed consider, for instance, the case where $\mathcal{S}=\{\{i, j\},\{i, k\}, N\}$ with $\theta_{j}<\theta_{k}$ and firm $i$ is the first firm to declare. If $w_{\alpha k}(i, k)$ is sufficiently large it could happen that $W_{\alpha i}(i, k)$ is strictly smaller than the greatest payoff firm $i$ can obtain in $\mathcal{V}_{\alpha}(N)$. Consequently, firm $i$ will propose the formation of the grand cartel and the best either firm $j$ or firm $k$ (or both) can do is to make a declaration compatible with that 
of firm $i^{8}$. This shows that the availability of a predatory strategy is not sufficient for predation to occur.

Finally, Part 3 of Proposition 1 corresponds to the example given in the Introduction above, except for the feasibility of the grand cartel. However, to have the same prediction, i.e. that the low marginal cost firm is excluded for all orders of declarations, two additional requirements are needed. The first is that the maximal capacity of firm $j$ is smaller than that of firm $i$; the second is that the maximal payoff that $j$ can obtain in the cartel $\{i, j\}$ is larger than the one it can obtain in cartel $(N)$. These conditions seem fairly unrestrictive: as firm $i$ has a lower marginal cost function than $j$ it is reasonable to assume that it has installed a higher capacity; while it is quite plausible that a firm can obtain more in a two-firm than in a threefirm cartel. obviously, if cartel $(N)$ was not feasible, as in the introductory example, then this second condition is trivially met.

As it can be seen from the Proof of Proposition 1 in the Appendix, these two conditions are superfluous for all orders of declaration except when $k$ is the first to declare. In this case $k$ in order to induce $j$ to enter the cartel $\{j, k\}$ must give to $j$ a payoff at least as great as the maximum payoff that $j$ could obtain in the cartel $\{i, j\}, W_{\alpha j}(i, j)$. The same is true if $k$ wants to induce $i$ to enter the cartel $\{i, k\}$, that is $k$ must give $W_{\alpha i}(i, j)$. Thus, $k$ will prefer the cartel $\{j, k\}$ if it gets a higher payoff in it rather than in $\{i, k\}$, given the constraints imposed by what he must offer to $j$ and $i$. This is the case if the conditions in Part 3 of Proposition 1 are met, as it is shown by Lemma 4 in the Appendix.

\section{Equilibria of the cartel formation game with costless reentry}

It has been shown in section 2.3, that the case with costless reentry differs from the one with unprofitable reentry only by the fact that in the former case the minimal payoff required by a firm, say $j$, to participate to a twofirms cartel, say $\{i, j\}$, is equal to $\max \left\{0, w_{\alpha j}(N)\right\}$ while in the latter case

\footnotetext{
${ }^{8}$ Note that if the cartel $\{j, k\}$ were also feasible then the grand cartel could form with firm $i$ being the first firm to declare and firm $j$ (resp. firm $k$ ) the second one provided that $W_{\alpha j}(j, k)$ (resp. $\left.W_{\alpha k}(j, k)\right)$ is strictly smaller than the highest payoff firm $j$ (resp. firm $k$ ) can obtain in $\mathcal{V}_{\alpha}(N)$.
} 
it equals $w_{\alpha j}(i, j)$. Accordingly if cartels $\{i, j\}$ and $\{i, k\}$ are feasible, that is, if $w_{\alpha j}(N)$ and $w_{\alpha k}(N)$ are negative, then the minimal payoff obtained by firms $j$ and $k$ in these cartels are equal to zero. Therefore, the highest payoff firm $i$ can obtain in a cartel, $W_{\alpha i}(s)$, depends on both the marginal and fixed costs of its partner. More precisely, let $q_{h}^{0}$ be the rival's output which leads to zero profit for firm $h$ when it plays its best reply, $q_{h}^{R}\left(q_{h}^{\circ}\right)$, that is, $q_{h}^{\circ}$ is such that $\pi_{h}\left(q_{h}^{R}\left(q_{h}^{0}\right), q_{h}^{0}\right)=0$. Furthermore, for all $q \in X_{h}^{0}$ with $X_{h}^{0}=\left[0, q_{h}^{0}\right)$, let $\hat{q}_{h}(q)$ be the smallest quantity produced by $h$ which gives it a zero profit whenever its rivals produce $q$, that is, $\hat{q}_{h}(q)$ is such that: $\pi_{h}\left(\hat{q}_{h}(q), q\right) \equiv 0$ and $\partial \pi_{h}\left(\hat{q}_{h}(q), q\right) / \partial q_{h}>0$. We have:

Lemma 2 Suppose all our assumptions except assumption 1 hold. There exists $\bar{\alpha}<1$ such that, if cartels $\{i, j\}$ and $\{i, k\}$ are feasible and $\left(\theta_{j}, F_{j}\right)$ and $\left(\theta_{k}, F_{k}\right)$ are such that $\hat{q}_{k}(q)>\hat{q}_{j}(q)$ for all $q \in X_{j}^{0} \cap X_{k}^{0}$, then $W_{\alpha i}(i, j)>$ $W_{\alpha i}(i, k)>W_{\alpha i}(N)$ for all $\alpha \in(\bar{\alpha}, 1)$.

Considering Figure 2, the proof of this result is clearly quite obvious and is thus omitted. It must be noticed that a necessary and sufficient condition for $W_{\alpha i}(i, j)>W_{\alpha i}(i, k)$ to hold would involve a comparison of the cost structure of the three firms. We thus choose to state our results in terms of a sufficient condition which actually requires only the comparison of firms $j$ and $k$ average cost function.

Clearly, Lemma 2 here will play the role of Lemma 1 in the case of noreentry. It therefore follows:

Proposition 2 Suppose all our assumptions except assumption 1 hold. There exists $\bar{\alpha}<1$ such that for all $\alpha \in(\bar{\alpha}, 1)$ and for any order of declaration we have:

1. A cartel forms,

2. let $\mathcal{S}=\{\{i, j\},\{i, k\}, N\}$ and $\hat{q}_{j}(q)<\hat{q}_{k}(q)$ for all $q \in X_{j}^{0} \cap X_{k}^{0}$, then cartels $\{i, k\}$ and $N$ do not form,

3. let $\mathcal{S}=\{\{i, j\},\{i, k\},\{j, k\}, N\}$ and $(i) \hat{q}_{i}(q)<\hat{q}_{j}(q)$ for all $q \in X_{i}^{0} \cap$ $X_{j}^{0}$, (ii) $\hat{q}_{i}(q)<\hat{q}_{k}(q)$ for all $q \in X_{i}^{0} \cap X_{k}^{0}$, (iii) $\hat{q}_{j}(q)<\hat{q}_{k}(q)$ for all $q \in X_{j}^{0} \cap X_{k}^{0}$ then cartels $\{i, k\},\{j, k\}$ and $N$ do not form. 
This Proposition ${ }^{9}$ contrasts with our previous results in two ways: First, the grand cartel, $N$, does not form, so that if seeing that there exists a predatory strategy it will be played i.e. predation occurs. This comes from the fact that, as long as cartels $\{i, j\}$ and $\{i, k\}$ are feasible, the minimal payoff firms $j$ and $k$ will obtain in both a two-firm cartel and in the grand cartel is equal to zero. It then follows that firm $i$ can always obtain a larger payoff in a two-firm cartel than in the grand cartel (see Lemma 3). Consequently if firm $i$ is the first firm to declare it will never propose the formation of the grand cartel. On the other hand if it is firm $j$ (resp. firm $k$ ) which is the first to declare then it will never propose the formation of the grand cartel. Indeed if it does so then both firm $i$ and firm $k$ (resp. firm $j$ ) can obtain a higher payoff than the one proposed in firm $j$ 's (resp. firm $k$ 's) declaration by making compatible declarations which propose the formation of the cartel $\{i, k\}$ (resp. $\{i, j\})$.

The second difference between the results with costless reentry and the ones with unprofitable reentry can be illustrated if we suppose that firms have identical fixed $\operatorname{costs}^{10}$. In this case $\hat{q}_{j}(q)<\hat{q}_{k}(q)$ for all $q \in X_{j}^{0} \cap X_{k}^{0}$ will hold if and only if $\theta_{j}<\theta_{k}$. Then Proposition 2 states simply that the firm with the highest marginal cost function will be predated. Therefore with costless reentry, contrary to what happens in the unpropfitable reentry case, a low marginal cost constitues a strong advantage to face predation. On the other hand, if we suppose that $\theta_{i}=\theta_{j}=\theta_{k}$ then the conditions used in Proposition 2 will be satisfied if and only if $F_{i}<F_{j}<F_{k}$. Hence we find back a result stated first by Ghemawat and Nalebuff (1985) for declining industries according to which the firm with the largest capacities i.e. with the highest fixed cost level is the first firm to exit the market. Such conclusion has also be drawn by Fudenberg and Tirole (1986) from the analysis of an incomplete information game.

To conclude with, if we are able to rank the firms with respect to their average cost function then Proposition 2 states that the exiting firm is the one with the highest average cost function.

\footnotetext{
${ }^{9}$ The proof of this Proposition follows so closely that of Proposition 1 that it s omitted.

${ }^{10}$ Recall that our results in the case of unprofitable reentry do not depend on the firms fixed costs.
} 


\section{Robustness of the results with unprofitable reentry}

One specific feature of the cartel formation game presented above is that each firm in its declaration proposes simultaneously a particular cartel and the payoffs that each member of the cartel will receive. As a consequence, the cartel formation game gives to all firms a strong influence on the way payoffs are allocated among cartel members.

This seems reasonable when reentry costs are negligible. In this case indeed the production game remains a three players game even if a firm exits the market. However when reentry costs are large, the production game becomes a two players game once a firm decides to stay out of the market. In this case one can ask the question if the cartel formation game does not give to the exiting firm an unrealistically excessive influence on the equilibrium of the resulting two firms production game which shall be played. In order to provide an answer, we shall analyze the sensitivity of the inefficiencies stated in Proposition 1 to the way firms are supposed to coordinate.

To investigate this issue we look at a two step coordination process where the exiting firm has no influence on the way the remaining firms will share the gains from cooperation in the production game. This coordination process constitutes a game: its first step is a substitute for the cartel formation game presented before. The only difference is that it is now supposed that a firm declaration only consists of a feasible cartel, s. If all declarations differ the game ends and each firm receives its reservation payoff $\bar{g}_{i}$. Otherwise one moves to the second step.

The second step consists of a negotiation between the members of the cartel given in the identical declarations of the first step, say s, to determine a payoff vector, $p$, belonging to $\mathcal{V}_{\alpha}(s)$. If a firm does not belong to $s$ then its action set in this step is simply $\{$ do nothing $\}$.

We shall not specify explicitely the bargaining game procedure. We as-

sume instead that, the gains from cooperation (i.e. the actual payoff minus the sum of appropriately discounted Cournot profits of the one-shot quantity game) are shared according to a bargaining solution. The bargaining solution we adopt here belongs to the family of egalitarian (also called proportional) 
solutions as axiomatized by Kalai (1977) and Kalai and Samet (1985) ${ }^{1112}$.

To be precise, let us first assume that:

Assumption 7 For any feasible cartel, s, the Cournot equilibrium in the quantity game is unique.

Then, let $\pi_{i}^{c}(i, j)$ denote the firm $i$ 's Cournot equilibrium profit when only firms $i$ and $j$ are active on the market. Furthermore denote by $\left(q_{i}^{e}(i, j), q_{j}^{e}(i, j)\right)$ the quantity vector which maximizes $P_{i}$ subject to $P_{i}-\pi_{i}^{c}(i, j)=P_{j}-\pi_{j}^{c}(i, j)$ and let $P_{\alpha i}^{e}(i, j)\left(\operatorname{resp} . P_{\alpha j}^{e}(i, j)\right)$ be given by $(1-\alpha) \sum_{t=0}^{\infty} \alpha^{t} \pi_{i}\left(q_{i}^{e}(i, j), q_{j}^{e}(i, j)\right)$ (resp. $\left.(1-\alpha) \sum_{t=0}^{\infty} \alpha^{t} \pi_{j}\left(q_{j}^{e}(i, j), q_{i}^{e}(i, j)\right)\right)$. Obviously $\left(P_{\alpha i}^{e}(i, j), P_{\alpha j}^{e}(i, j)\right)$ is the symmetric egalitarian solution ${ }^{13}$ to the cooperative bargaining game defined by a set of outcomes given by $\mathcal{F}(i, j)$ and a statu-quo point given by $\left(\pi_{i}^{c}(i, j), \pi_{j}^{c}(i, j)\right)$.

We can immediately state:

Lemma 3 Let all our assumptions except 2 be satisfied. Furthermore, for any feasible two-firms cartel, say $\{h, l\}$, suppose that $\left(q_{h}^{e}(h, l), q_{l}^{e}(h, l)\right)$ belongs to $] 0, \bar{q}_{h}[\times] 0, \bar{q}_{l}\left[\right.$ and that there exits $\left(q_{h}, q_{l}\right) \gg 0$ which maximizes $P_{h}+P_{l}$. Then there exists $\bar{\alpha}<1$ such that, for all $\alpha>\bar{\alpha}, P_{\alpha i}^{e}(i, k)>P_{\alpha i}^{e}(i, j)$ if and only if $\theta_{k}>\theta_{j}$.

\footnotetext{
${ }^{11}$ This kind of structure has already been used in the literature. For instance, in Grossman and Hart (1986), two agents first choose non-cooperatively and simultaneously a level of investment and then, given these investments, take actions such that the gains from renegotiation, which correspond to the gains from cooperation in our framework, is shared equally. In their context, this corresponds also to the Nash bargaining solution. The Groosman and Hart's analysis has been extended by Hart and Moore (1990) to many agents and the bargaining solution adopted there to share the gain from trade is the Shapley value.

We adopt here an egalitarian solution one the one hand because it is much more tractable than the other ones (in particular the Nash bargaining solution), and on the other hand because the egalitarian solutions are the only ones which, in the presence of other standard requirements, satisfy the monotonicity property (see Kalai and Samet (1985)). This condition simply states that if the feasible set of one coalition increases and the feasible sets of all other coalitions remain the same, then none of the members of this coalition should become worse off because of this change.

${ }^{12}$ Note that similar results could be obtained by using the symmetric Nsah bargaining solution.

${ }^{13}$ It will be obvious to verify that the results presented below will hold if we take an asymmetric egalitarian solution provided the weight of firm $i$ in the solution depends negatively on $\theta_{i}$ and is independent on the fixed costs level.
} 
Notice that the first additional assumption in this Lemma will simply guarantee that there exists a feasible payoff vector strictly greater than the Cournot equilibrium profits vector.

Again an increase in $\theta_{j}$ will have two effects on the cooperative bargaining game involving firms $i$ and $j$ : On the one hand, it leads to a modification in the set of feasible outcomes which affects negatively the payoff of firm $i$ at the egalitarian solution while, on the other hand, it increases (resp. decreases) firm $i$ 's (resp. firm $j$ 's) statu-quo payoff which will rise the firm $i$ 's payoff at the egalitarian solution. The Lemma ${ }^{14}$ states simply that the positive effect arising from the move in the statu-quo payoff dominates the negative effect coming from the reduction in the set of feasible outcomes.

This result will play, for Proposition 3 below, the role played by Lemma 1 and 2 for Proposition 1 and 2 respectively. To see this it suffices to realize that the set of subgame perfect equilibria of the game deriving from the two step procedure here considered coincides with the one of the cartel formation game where a firm $i$ 's declaration consists of a feasible cartel, $s_{i}$, to which firm $i$ belongs and of a payoff vector which gives to each firm in $s_{i}$ the symmetric egalitarian payoff defined above ${ }^{15}$ and a zero payoff to a firm (if any) which does not belong to $s_{i}$. Formally the set of firm $i$ 's declarations is now $\mathcal{D}_{i}=$ $\left\{(s, p) \mid i \in s, s \in \mathcal{S}\right.$, for all $h \in s p_{h}=p_{h}^{e}(s)$ and, for $\left.l \notin s, p_{l}=0\right\}$. Therefore we have:

Proposition 3 Let all assumptions in Lemma 3 hold. Then there exists $\bar{\alpha}<1$ such that for all $\alpha \in(\bar{\alpha}, 1)$ and whatever the order of declaration we have:

1. A cartel forms,

2. if $\mathcal{S}=\{\{i, j\},\{i, k\}, N\}$ and $\theta_{j}<\theta_{k}$, then cartel $\{i, j\}$ does not form,

3. if $\mathcal{S}=\{\{i, j\},\{i, k\},\{j, k\}, N\}$ and $\theta_{i}<\theta_{j}<\theta_{k}$, then cartels $\{i, j\}$ and $\{i, k\}$ do not form.

\footnotetext{
${ }^{14}$ The proof of this result comes quite straightforwardly from the application of the envelope theorem as well as the Folk theorem. Hence it will be omitted.

${ }^{15}$ To save space we do not define formally the egalitarian payoff when the three firms are active. However this can easily be done even if one wants to consider a coalition form game instead of a cooperative bargaining game. Anyway this does not matter for our analysis.
} 
This shows the robustness of our conclusions with respect to the influence of the exiting firm on the way payoffs are allocated in the production game.

\section{Concluding remarks}

We have considered in this paper a dynamic production game involving three firms which are differentiated according to their cost function. More precisely we have assumed that firms can be ranked unambiguously according to their marginal cost function and that their fixed cost may differ. Furthermore we suppose that one firm can be credibly forced to stay out of the market by the two others and that at least two firms can be put under such a threat. We then investigate the cost characteristics of the exiting firm under two alternative hypothesis concerning the possibility of reentry namely the case where reentry is unprofitable in any circumstances due to the presence of large sunk costs, and the one where reentry is costless.

We have obtained two predictions (which appears quite robust to the specification of the cartel formation game). First if reentry is always unprofitable then the exiting firm has the lowest marginal cost function as compared with the marginal cost function of the firms which can credibly be predated. Furthermore this result does not depend on the level of fixed $\operatorname{costs}^{16}$. Accordingly, in this case, cost inefficiencies will arise since the exiting firm is the one which uses the most efficient technology.

A second result is that when reentry is costless and when we can rank firms according to their average cost function then the exiting firm has the larger average cost function as compared to the average cost function of the firms which can be put under the threat of predation. Therefore in this case cost inefficiencies do not appear.

The result obtained in the no-reentry case looks strange since it goes against the common belief that the most efficient firm will remain on the market. But this belief has been developped in the context of "neo-classical economics". If instead we look at this result from the point of view of "transaction cost economics" (as developped in Williamson (1985) for instance) then they appear rather unsurprising. Indeed in this context such kind of inefficiencies are frequently obtained. It is worthwhile emphasizing the deep

\footnotetext{
${ }^{16}$ However the set of firms which can be predated depend obviously on the level of fixed costs.
} 
relationship between our analysis and the transaction cost approach. Indeed although the latter approach focuses mainly on the internal organisation of the firm the present study shows that the basic points which distinguish transaction cost economics from other economic approches are also well suited to study the composition of an industry and more generally to make substantial progresses in the understanding of the formation and composition of groups or coalitions on a market.

Roughly speaking transaction cost economics seeks to analyse situations involving agents characterized by opportunism and bounded rationality where (i) agents will meet frequently, (ii) agents do not rely on courts for settling disputes among them i.e. private ordering prevails, (iii) agents have the opportunity to make asset specific investments and (iv) agents evolve in an uncertain environment.

In the present analysis we have ruled out both uncertainty and bounded rationality since these characteristics appear unessential for our results. Note furthermore that frequency will not be relevant here as the example given in the Introduction points out. The difference between opportunism and self-interested behavior does not matter here because the set of subgame perfect Nash equilibria and the set of Nash equilibria of the production game coincides for a discount factor sufficiently close to zero. We shall however argue that if we make abstraction of the presence of either private ordering or asset specific investments then the cost inefficiencies obtained in the paper disappear.

Let us begin with private ordering. Many exchange analysis suppose that efficacious rules of law are in place so that any disagreement regarding the execution of a contract is settled by courts in a fully informed and low-cost way. This assumption of court ordering is very convenient since it allows to disregard the ex-post side of a contract. In our context, firms cannot rely on court since the kind of contract they are willing to do is simply illegal. An immediate consequence of private ordering is that we cannot disregard the execution phase of the contract since the latter must be self enforcing. This entails that firms, as is supposed in the cartel formation game, will only consider payoff vectors which can be associated with a subgame perfect Nash equilibrium of the production game.

But suppose to the contrary that firms can rely costlessly on court to enforce an agreement. This implies that the set of payoff vectors that must now be considered in the cartel formation game coincides with the one cor- 
responding to the costless reentry case. Indeed, a firm which can be forced to exist can commit to obey an agreement in which it receives a zero payoff. Without this possibility of commitment, such an agreement is not credible in the no-reentry case while it is in the case of costless reentry. Consequently, the result stated in Lemma 2 will hold even if reentry is unprofitable and the exiting firm is the one with the highest average cost function (see Proposition 2 for a more precise statement). Therefore the cost inefficiencies disappear once court ordering is allowed for. Remark that this clearly shows that considering tacit cooperation between firms as illegal is possibly costly.

Let us now turn to the asset specific character of investments. Investments are said wholly asset specific if they are unredeployable. Accordingly investment costs are sunk for wholly asset specific investments while they are fixed when investment looses its asset specific character. The main consequence of the presence of asset specific investment is the occurence of the fundamental transformation. The latter concept refers to the transformation in the nature of the competition prevailing before and after the adoption of the contract.

In our context, the sunk reentry cost we have introduced can simply be interpreted as the cost of unredeployable investments. More precisely, the unprofitable reentry case corresponds to the situation where large asset specific investments must be achieved before being active on the market while in the costless reentry case such investments are negligible. When reentry is unprofitable the fundamental transformation occurs since, once a firm exits, the production game becomes a two players game. If instead reentry is costless this transformation does not occur. Indeed, in this case even if a firm exits it can participate to the punishment of a deviation from the equilibrium path by one of the two firms which remain on the market. In other words the production game still involves three players even if a firm exits the market. As we have shown, cost inefficiencies appear only in the case of unprofitable reentry which means that the presence of large asset specific investments is a necessary condition for such cost inefficiencies to occur. 


\section{References}

Aumann, R. and R. Myerson 1988: "Endogenous formation of links between players and coalitions: An application of the Shapley value". In: The Shapley Value: Essays in Honor of Lloyd Shapley, edited by A. Roth. Cambridge University Press, Cambridge, U.K.

Bernheim, D., D. Peleg and M. Whinston 1987: "Coalition-proof Nash equilibria. I: Concepts". Journal of Economic Theory, vol 42 pages112 .

Bloch, F.1990a: "Sequential formation of coalitions and stable coalition structures in symmetric games". Mimeo, Department of Economics, University of Pennsylvania.

Bloch, F.1990b: "Endogenous structures of associations in oligopolies". Mimeo, Department of Economics, University of Pennsylvania.

Fudenberg, D. and J. Tirole 1986: "A theory of exit in duopoly". Econometrica vol 54 pages 943- 960 .

Ghemawat, P. and G. Nalebuff 1985: "Exit". Rand Journal of Economics vol 16 pages 184-194.

Ghemawat, P. and G. Nalebuff 1990: "The evolution of declining industries". Quaterly Journal of Economics vol 105 pages 167-186.

Grossman, S. and O. Hart 1986: "The costs and Benefits of ownership: A theory of vertical and lateral integration". Journal of Political Economy vol 94, pages 691-719.

Gul, F.1989: "Bargaining foundations of Shapley value", Econometrica vol 57 pages $81-95$.

Hart, O. and J. Moore 1990: "Property rights and the nature of the firm". Journal of Political Economy vol 98 pages 1119-1158.

Kalai, E.1977: "Proportional solutions to bargaining situations: Interpersonal utility comparisons". Econometrica vol 45 pages 1623-1630.

Kalai, E. and D. Samet 1985: "Monotonic solutions to general cooperative games". Econometrica vol 53 pages 307-327.

Tirole J. 1988: The Theory of Industrial Organization. MIT Press, Cambridge Mass. 
Williamson, O. 1985 The Economic Institutions of Capitalism. The Free Press New-York.

Wilson, R. 1992: "Strategic models of entry deterrence". In: Handbook of Game Theory ed. by Aumann, R. and S. Hart, North-Holland/ Elsevier Science Publishers. Amsterdam. 


\section{Appendix}

\section{Proof of Proposition 1}

\subsection{A cartel forms.}

The proof that a cartel forms is straightforward and it is therefore omitted.
8.2 Let $\mathcal{S}=\{\{i, j\},\{i, k\}, N\}$ and $\theta_{j}<\theta_{k}$, then cartel $\{i, j\}$ does not form.

Remark immediately that since $\{i, j\}$ and $\{i, k\}$ are both feasible we have $\bar{g}_{j}=\bar{g}_{k}=0$. Obviously, it is useless to consider strategies that lead to the formation of cartel $N$ since we are only interested to prove that cartel $\{i, j\}$ does not form.

\subsubsection{Suppose that firm $i$ is the first firm to declare:}

1. (a) Let firm $k$ be the last firm to declare. If $d_{i} \notin \mathcal{D}_{k}$ (i.e. the proposed cartel in the firm $i$ 's declaration is the cartel $\{i, j\}$ ) then, whatever is firm $j$ 's declaration and whatever is its own declaration firm $k$ will obtain a zero payoff (either $d_{i}=d_{j}$ and in this case firm $k$ obtains $p_{i k}=p_{j k}=0$ since $p_{i} \in \mathcal{V}_{\alpha}(i, j)$ implies $p_{i k}=0$ or $d_{i} \neq d_{j}$ in which case firm $k$ will obtain $\bar{g}_{k}=0$ ). Now if $d_{i} \in \mathcal{D}_{k}$ we must distinguish two cases: On the one hand, if $d_{i} \notin \mathcal{D}_{j}$ (i.e. firm $i$ proposes the formation of cartel $\{i, k\}$ ) then firm $k$ will obtain a zero payoff by declaring $d_{k} \neq d_{i}$ while it will receive $p_{i k} \geq w_{\alpha k}(i, k)>0$ by declaring $d_{k}=d_{i}$. On the other hand if $d_{i} \in \mathcal{D}_{j} \cap \mathcal{D}_{k}$ then firm $k$ will obtain $p_{i k} \geq 0$ ( since $\left.w_{\alpha k}(N)<0\right)$ if either it declares $d_{k}=d_{i}$ or $d_{i}=d_{j}$ while it will receive $\bar{g}_{k}=0$ if $d_{i} \neq d_{j} \neq d_{k}$. It follows that all firm $k$ 's best-response to $\left(d_{i}, d_{j}\right)$ is characterized by: (i) $d_{k}$ can be anything if either $d_{i} \notin \mathcal{D}_{k}$ or $d_{i}=d_{j}$ or $p_{i k}=0$; (ii) $d_{k}=d_{i}$ if $d_{i} \in \mathcal{D}_{k}$ and $p_{i k}>0$ and $d_{j} \neq d_{i}$. 
(b) Remark that if firm $j$ were the last firm to declare a similar reasoning will lead to the conclusion that all firm $j$ 's best-response to $\left(d_{i}, d_{k}\right)$ will satisfy (i) $d_{j}$ can be anything if either $d_{i} \notin \mathcal{D}_{j}$, or $d_{i}=d_{k}$, or $p_{i j}=0$; (ii) $d_{j}=d_{i}$ if $d_{i} \in \mathcal{D}_{j}$ and $p_{i j}>0$ and $d_{k} \neq d_{i}$.

2. (a) Let us consider the problem faced by the second firm to declare, say firm $j$. If $d_{i} \notin \mathcal{D}_{j}$ then, whatever its own declaration is, $j$ will receive a zero payoff. Given point 1.(b) above, if $d_{i} \in \mathcal{D}_{j} \backslash \mathcal{D}_{k}$ (i.e. firm $i$ proposes the formation of cartel $\{i, j\}$ ) then it will obtain a payoff equal to $p_{i j} \geq w_{\alpha j}(i, j)$ if it declares $d_{j}=d_{i}$ or $\bar{g}_{j}=0$ if it declares $d_{j} \neq d_{i}$. If $d_{i} \in \mathcal{D}_{j} \cap \mathcal{D}_{k}$ (i.e. firm $i$ proposes the formation of cartel $N$ ) then, since firm $k$ best-response to $\left(d_{i}, d_{j}\right)$, with $d_{j} \neq d_{i}$ is $d_{k}=d_{i}, j$ will obtain $p_{i j} \geq 0$ either by declaring $d_{j}=d_{i}$ or by declaring anything else. On the other hand if $d_{i} \in \mathcal{D}_{j} \cap \mathcal{D}_{k}$ and $d_{i}$ is such that firm $k$ 's best-response is $d_{k} \neq d_{i}$ for $d_{j} \neq d_{i}$ then firm $j$ will obtain a zero payoff if it declares $d_{j} \neq d_{i}$ while it receives $p_{i j} \geq 0$ if it declares $d_{j}=d_{i}$.

Therefore all firm $j$ 's best-responses to $d_{i}$ will satisfy: (i) $d_{j}$ can be anything if either $d_{i} \notin \mathcal{D}_{j}$ or $p_{i j}=0$, or $d_{i}$ is such that firm $k$ 's best-response to $\left(d_{i}, d_{j}\right)$, with $d_{j} \neq d_{i}$, is $d_{k}=d_{i}$; (ii) $d_{j}=d_{i}$ if $d_{i} \in \mathcal{D}_{j}$, and $p_{i j}>0$, and $d_{i}$ is such that firm $k$ 's best-response to $\left(d_{i}, d_{j}\right)$, with $d_{j} \neq d_{i}$, is $d_{k} \neq d_{i}$.

(b) If firm $k$ were the second firm to declare a similar reasoning will lead to the same characterization of all firm $k$ 's best-response to $d_{i}$.

3. Now consider the problem faced by firm $i$.

First, if it declares $d_{i}^{j} \equiv\left(\{i, j\},\left(W_{\alpha i}(i, j), w_{\alpha j}(i, j), 0\right)\right)$, firm $j$ will declare $d_{j}=d_{i}^{j}$ (since $d_{i}^{j} \notin \mathcal{D}_{k}$ ) and consequently firm $i$ will obtain $W_{\alpha i}(i, j)$. Second if it declares $d_{i}^{k} \equiv\left(\{i, k\},\left(W_{\alpha i}(i, k), 0, w_{\alpha k}(i, k)\right)\right)$ firm $k$ will declare $d_{k}=d_{i}^{k}$ whatever the decalration of firm $j$ (since $d_{i}^{k} \notin \mathcal{D}_{j}$ and $\left.p_{i k}>0\right)$ and firm $i$ will obtain $W_{\alpha i}(i, k)$. Third if firm $i$ declares $d_{i}^{N}=\left(N,\left(P_{i}(\epsilon), \epsilon, 0\right)\right)$, for some $\epsilon>0$ and $P_{i}(\epsilon)$ being the maximal payoff firm $i$ can obtain in $\mathcal{V}_{\alpha}(N)$ when $P_{j}=\epsilon$ and $P_{k}=0$, then firm $j$ will declare $d_{j}=d_{i}^{N}$ and it follows that firm $i$ will receive $P_{i}(\epsilon)$. Remark immediately that $P_{i}(\epsilon)$ tends to $W_{\alpha i}(N)$ when $\epsilon$ tends to zero. Finally if firm $i$ makes a declaration, $d_{i}^{O}$, leading firm $j$ to 
declare $d_{j} \neq d_{i}^{O}$ and firm $k$ to declare $d_{k} \neq d_{i}^{O}$ then firm $i$ will receive $\bar{g}=w_{\alpha i}(N)$.

Firm $i$ will never make a declaration like $d_{i}^{O}$ since doing so it will receive $w_{\alpha i}(N)$ which is strictly smaller than the payoff it will obtain if it decalares $d_{i}^{j}$ for instance. Furthermore since $\theta_{j}<\theta_{k}$ we know by Lemma 1 that $W_{\alpha i}(i, k)>W_{\alpha i}(i, j)$ for $\alpha$ sufficiently close to one. Therefore firm $i$ will never declare $d_{i}^{j}$ and the cartel $\{i, j\}$ does not form.

\subsubsection{Suppose that firm $i$ is the second firm to declare:}

1. Let firm $k$ be the last firm to declare. Any best-response for this firm satisfies the properties given in point B.2. 1a. It follows that by declaring $d_{i}^{k}$, defined in point B.2.1 3 , firm $i$ will obtain $W_{\alpha i}(i, k)$. Consequently for firm $i$ to maximize its payoff by declaring $d_{i}=d_{j}$ it is necessary that $p_{j i} \geq W_{\alpha i}(i, k)$. Therefore all firm $i$ 's best-responses to $d_{j}$ are such that: (i) $d_{i}=d_{i}^{k}$ if $p_{j i}<W_{\alpha i}(i, k)$; (ii) $d_{i}=d_{j}$ if $p_{j i}>W_{\alpha i}(i, k)$. Now, by Lemma 1 , for $\alpha$ sufficiently close to one there does not exist $d_{j} \in \mathcal{D}_{j}$ such that $d_{j} \notin \mathcal{D}_{k}$ and $p_{j i} \geq W_{\alpha i}(i, k)$. This implies that cartel $\{i, j\}$ does not form.

2. Let firm $j$ be the last firm to declare. Reasoning as above, it follows that all firm $i$ 's best-responses to $d_{k}$ are such that: (i) $d_{i}=d_{i}^{j}$ if $p_{k i}<W_{\alpha i}(i, j)$; (ii) $d_{i}=d_{k}$ if $p_{k i}>W_{\alpha i}(i, j)$. But for $\alpha$ sufficiently close to one, Lemma 1 ensures the existence of $d_{k} \in \mathcal{D}_{k}$ such that $p_{k i}>W_{\alpha i}(i, j)$. Moreover, by making a declaration $d_{k} \notin \mathcal{D}_{j}$, which induces firm $i$ to declare $d_{i}=d_{k}$, firm $k$ will obtain a strictly greater payoff than the one it will obtain by declaring any other $d_{k} \in \mathcal{D}_{k}$. Therefore cartel $\{i, j\}$ does not form.

\subsubsection{Suppose that firm $i$ is the last firm to declare:}

1. If $d_{j}=d_{k}$ (i.e. cartel $N$ is proposed) then firm $i$ will obtain $p_{j i}=p_{k i}$ whatever is its own declaration. If $d_{j} \neq d_{k}$ then firm $i$ will obtain either $\bar{g}_{i}=w_{\alpha i}(N)$ by declaring $d_{i}$ which differs from both $d_{j}$ and $d_{k}$, or $p_{j i}$ by declaring $d_{i}=d_{j}$, or $p_{k i}$ by declaring $d_{i}=d_{k}$. But $p_{j i} \geq w_{\alpha i}(N)$ and $p_{k i} \geq w_{\alpha i}(N)$. It follows that all firm $i$ 's best-responses to $\left(d_{j}, d_{k}\right)$ 
satisfy: (i) $d_{i}$ can be anything if $d_{j}=d_{k}$; (ii) $d_{i}=d_{j}$ if $p_{j i}>p_{k i}$; (iii) $d_{i}=d_{k}$ if $p_{k i}>p_{j i}$.

2. Let firm $k$ be the second firm to declare. If $d_{j} \notin \mathcal{D}_{k}$, i.e. firm $j$ proposes the formation of cartel $\{i, j\}$, then firm $k$ will receive a zero payoff if it makes a declaration such that the best-response to $\left(d_{j}, d_{k}\right)$ is $d_{i}=d_{j}$. However, for $\alpha$ sufficiently close to one, Lemma 1 ensures that, for any $d_{j} \notin \mathcal{D}_{k}$, there exists a $d_{k} \in \mathcal{D}_{k}$ with $p_{k i}>p_{j i}$ and $p_{k k}>w_{\alpha k}(i, k)>p_{j k}=0$. Therefore cartel $\{i, j\}$ does not form.

3. Let firm $j$ be the second firm to declare. Firm $k$ receives zero if $\{i, j\}$ forms. This, however, can be prevented by firm $k$ : from Lemma 1 , it can make a declaration with $p_{k i}>W_{\alpha i}(i, j)$ and $s=\{i, k\}$. Therefore, since a cartel forms and using subgame perfection, $\{i, j\}$ cannot form.

1.3 Let $\mathcal{S}=\{\{i, j\},\{i, k\},\{j, k\}, N\}$ and $\theta_{i}<\theta_{j}<\theta_{k}$, then cartel $\{i, j\}$ does not form. Furthermore if in addition $\bar{q}_{j} \leq \bar{q}_{i}$ and $W_{\alpha j}(i, j) \geq$ $W_{\alpha j}(N)$ then cartels $\{i, j\}$ and $\{i, k\}$ do not form.

Remark immediately since $\{i, j\},\{i, k\}$ and $\{j, k\}$ are simultaneously feasible we have $\bar{g}_{i}=\bar{g}_{j}=\bar{g}_{k}=0$.

To begin with let us characterize the best-responses of the last firm to declare. For the generality of the argument we shall index the last firm by $o$ and the two others by $m$ and $n$. First of all, if $d_{m}=d_{n}$ then whatever its declaration firm $o$ will receive $p_{m o}$. Second, if $d_{m} \neq d_{n}$ and $d_{m} \notin \mathcal{D}_{o}$ and $d_{n} \notin \mathcal{D}_{o}$, then whatever its declaration firm $o$ will receive a zero payoff. Finally, suppose that $d_{m} \neq d_{n}$ and either $d_{m} \in \mathcal{D}_{o}$ or $d_{n} \in \mathcal{D}_{0}$ or both, then firm $o$ will obtain either $p_{m o}$ if $d_{m} \in \mathcal{D}_{o}$ and it declares $d_{o}=d_{m}$, or $p_{n o}$ if $d_{n} \in \mathcal{D}_{o}$ and it declares $d_{o}=d_{n}$, or zero if it makes a declaration which differs simultaneously from $d_{m}$ and $d_{n}$. Accordingly, all firm $o$ 's best-responses to $\left(d_{m}, d_{n}\right)$ satisfy: (i) $d_{o}$ can be anything if either $d_{m}=d_{n}$ or $d_{m} \neq d_{n}, d_{m} \notin \mathcal{D}_{0}$ and $d_{n} \notin \mathcal{D}_{o}$ or $p_{m o}=p_{n o}=0$; (ii) $d_{o}=d_{m}$ if $d_{m} \in \mathcal{D}_{0}$ and $p_{m o}>p_{n o}$; (iii) $d_{o}=d_{n}$ if $d_{n} \in \mathcal{D}_{0}$ and $p_{n o}>p_{m o}$. 


\subsubsection{Suppose that firm $k$ is the last firm to declare:}

1. Let firm $j$ be the second firm to declare. For the cartel $\{i, j\}$ to form it must be the case that $d_{i}=d_{j}, d_{i} \in \mathcal{D}_{j} \backslash \mathcal{D}_{k}$. But if firm $i$ makes a declaration belonging to $\mathcal{D}_{j} \backslash \mathcal{D}_{k}$, firm $j$ will obtain at most $W_{\alpha j}(i, j)$ by declaring $d_{j}=d_{i}$ while it will receive $W_{\alpha j}(j, k)$ if it declares $d_{j}^{k}=$ $\left(\{j, k\},\left(0, W_{\alpha j}(j, k), w_{\alpha k}(j, k)\right)\right)$, since for such declarations $\left(d_{i}, d_{j}^{k}\right)$ firm $k$ will maximize its payoff by declaring $d_{k}=d_{j}^{k}$. By Lemma 1 we know that, for $\alpha$ sufficiently close to one, $W_{\alpha j}(j, k)>W_{\alpha j}(i, j)$ and therefore cartel $\{i, j\}$ does not form.

For cartel $\{i, k\}$ to form it must be the case that $d_{i} \notin \mathcal{D}_{i} \cap \mathcal{D}_{j}$. If firm $j$ makes a declaration which induces firm $k$ to declare $d_{k}=d_{i}$ then it will receive a zero payoff. However for any $p_{i k} \in\left[w_{\alpha k}(i, k), W_{\alpha k}(i, k)\right]$ we know by Lemma 1 that, for $\alpha$ sufficiently close to one, there exists $d_{j} \in \mathcal{D}_{j} \cap \mathcal{D}_{k}$ such that $p_{j k}>p_{i k}$ and $p_{j j}>w_{\alpha j}(j, k)>0$. Therefore cartel $\{i, k\}$ does not form.

2. Let firm $i$ be the second firm to declare. For the cartel $\{i, j\}$ to form it must be the case that $d_{i}=d_{j}, d_{j} \in \mathcal{D}_{i} \backslash \mathcal{D}_{k}$. But if firm $j$ makes such a declaration firm $i$ will receive at most $W_{\alpha i}(i, j)$ by declaring $d_{i}=d_{j}$ while it will receive $W_{\alpha i}(i, k)$ if it declares $d_{i}^{k}=$ $\left(\{i, k\},\left(W_{\alpha i}(i, k), 0, w_{\alpha k}(i, k)\right)\right)$ since for such $\left(d_{j}, d_{i}^{k}\right)$ firm $k$ will maximize its payoff by declaring $d_{k}=d_{i}^{k}$. By Lemma 1, for $\alpha$ sufficiently close to one, $W_{\alpha i}(i, k)$ is stricly greater than $W_{\alpha i}(i, j)$ and therefore cartel $\{i, j\}$ does not form.

For cartel $\{i, k\}$ to form it must be the case that $d_{i} \in \mathcal{D}_{k} \backslash \mathcal{D}_{j}$ and $d_{i} \notin \mathcal{D}_{j} \cap \mathcal{D}_{k}$ and $d_{j}$ is such that $p_{i k} \geq p_{j k}$ (the equality between $p_{i k}$ and $p_{j k}$ is allowed only if firm $k$, facing two indifferent alternatives, chooses to declare $d_{k}=d_{j}$ ). In this situation firm $j$ will obtain a zero payoff while firm $k$ will obtain at most $W_{\alpha k}(i, k)$. However, by Lemma 1 , we know that, for $\alpha$ sufficiently close to one, there exists $d_{j} \in \mathcal{D}_{j} \cap \mathcal{D}_{k}$ such that $p_{j k}>W_{\alpha k}(i, k)$ and $p_{j j}>w_{\alpha j}(j, k)>0$. Consequently cartel $\{i, k\}$ does not form.

Summing up, if $k$ is the last firm to declare, Lemma 1 is sufficient to ensure that the cartel which form is either $\{j, k\}$ or $N$. 


\subsubsection{Suppose that firm $j$ is the last firm to declare:}

1. Let firm $k$ be the second firm to declare. For the cartel $\{i, j\}$ to form it must be the case that $d_{i} \in \mathcal{D}_{j} \backslash \mathcal{D}_{k}$ and $d_{k}$ is such that $p_{k j} \leq p_{i j}$ (the equality between $p_{k j}$ and $p_{i j}$ is allowed only if firm $j$, facing two indifferent alternatives, chooses to declare $d_{j}=d_{i}$ ). In this case firm $j$ will obtain at most $W_{\alpha j}(i, j)$ while firm $k$ will obtain a zero payoff. However, by Lemma 1 , if $\alpha$ is sufficiently close to one then for any $d_{i}$ which does not belong to $\mathcal{D}_{i} \cap \mathcal{D}_{k}$ there exists $d_{k} \in \mathcal{D}_{j} \cap \mathcal{D}_{k}$ such that $p_{k j}>p_{i j}$ and $p_{k k}>w_{\alpha k}(j, k)>0$. Therefore cartel $\{i, j\}$ does not form.

For cartel $\{i, k\}$ to form it must be the case that $d_{i} \in \mathcal{D}_{k} \backslash \mathcal{D}_{j}$ and $d_{i}=d_{k}$. But since $d_{i} \notin \mathcal{D}_{j}$ firm $j$ will maximize its payoff by declaring $d_{j}=d_{k}$ as long as $d_{k} \in \mathcal{D}_{k}$ and $p_{k j}>0$. Therefore once $d_{i} \notin \mathcal{D}_{i} \cap \mathcal{D}_{j}$ firm $k$ can obtain a payoff of at least $W_{\alpha k}(j, k)$ while it obtains at most $W_{\alpha k}(i, k)$ by declaring $d_{k}=d_{i}$. By Lemma 1 , for $\alpha$ sufficiently close to one, $W_{\alpha k}(i, k)<W_{\alpha k}(j, k)$ and consequently cartel $\{i, k\}$ does not form.

2. Let firm $i$ be the second firm to declare. Define $\mathcal{D}_{i}^{R}\left(d_{k}\right)=\left\{d_{i} \in\right.$ $\mathcal{D}_{i} \mid p_{i i} \geq p_{k i}$ and $\left(d_{i}, d_{k}\right)$ is such that firm $j$ 's best-response is $d_{j}=$ $\left.d_{i}.\right\}$. For cartel $\{i, j\}$ to form a necessary condition is that firm $k$ makes a declaration such that $\mathcal{D}_{i}^{R}\left(d_{k}\right) \backslash \mathcal{D}_{k} \neq \emptyset$. But if firm $k$ makes such a declaration it will obtain a zero payoff while we know by Lemma 1 that, for $\alpha$ sufficiently close to one, there exists $d_{k} \in \mathcal{D}_{k} \backslash \mathcal{D}_{i}$ such that it obtains a payoff strictly greater than $w_{\alpha k}(j, k)>0$ and for which $\mathcal{D}_{i}^{R}\left(d_{k}\right) \backslash \mathcal{D}_{k}=\emptyset$. Consequently cartel $\{i, j\}$ does not form.

Now for cartel $\{i, k\}$ to form it is necessary that firm $k$ makes a declaration such that $d_{k} \notin \mathcal{D}_{j}$ and $p_{k i} \geq \max \left\{W_{\alpha i}(i, j), W_{\alpha i}(N)\right\}$. Indeed if $d_{k} \notin \mathcal{D}_{j}$ then firm $j$ will declare $d_{j}=d_{i}$ as long as $d_{i} \in \mathcal{D}_{j}$ and $p_{i j}>0$. Accordingly for $d_{k}$ such that $d_{k} \notin \mathcal{D}_{j}$ firm $i$ can obtain either $W_{\alpha i}(i, j)$ by declaring: $d_{i}^{j}=\left(\{i, j\},\left(W_{\alpha i}(i, j), w_{\alpha j}(i, j), 0\right)\right)$ or the maximal payoff, denoted by $P_{i}^{N}(\epsilon)$, that firm $i$ can obtain in $\mathcal{V}_{\alpha}(N)$ when $P_{j}=\epsilon>0$ and $P_{k}=0$, by declaring $d_{i}^{N}=\left(N,\left(P_{i}^{N}(\epsilon), \epsilon, 0\right) . P_{i}^{N}(\epsilon)\right.$ tends to $W_{\alpha i}(N)$ when $\epsilon$ tends to zero. It follows that if $d_{k} \notin \mathcal{D}_{j}$ and $p_{k i}<\max \left\{W_{\alpha i}(i, j), W_{\alpha i}(N)\right\}$ then firm $i$ will maximize its payoff by declaring either $d_{i}^{j}$ or $d_{i}^{N}$ and cartel $\{i, k\}$ does not form. Con- 
sequently if $W_{\alpha i}(i, k)<W_{\alpha i}(N)$ cartel $\{i, k\}$ does not form while if $W_{\alpha i}(i, k) \geq W_{\alpha i}(N)$ the maximal payoff firm $k$ can obtain when cartel $\{i, k\}$ forms is equal or smaller than $\tilde{w}_{\alpha k}(i, k)$.

On the other hand for cartel $\{j, k\}$ to form it is sufficient that firm $k$ 's declaration be such that $d_{k} \notin \mathcal{D}_{i}$ and $p_{k j}>\max \left\{W_{\alpha j}(i, j), W_{\alpha j}(N)\right\}$. Indeed for such firm $k$ 's declaration there does not exist $d_{i} \in \mathcal{D}_{i}$ such that $p_{i j} \geq p_{k j}$ and thus firm $j$ will declare $d_{j}=d_{k}$. The assumption that $W_{j}(i, j) \geq W_{j}(N)$ together with Lemma 1 ensure that, for $\alpha$ sufficiently close to one, we have $W_{\alpha j}(j, k)>\max \left\{W_{\alpha j}(i, j), W_{\alpha j}(N)\right\}=W_{\alpha j}(i, j)$. Hence there exists $d_{k} \in \mathcal{D}_{k} \backslash \mathcal{D}_{i}$ such that $p_{k j}>\max \left\{W_{\alpha j}(i, j), W_{\alpha j}(N)\right\}$. Futhermore, for $\alpha$ sufficiently close to one, we also have by Lemma 4 that $\tilde{w}_{\alpha k}(i, k)<\tilde{w}_{\alpha k}(j, k)$. This implies the existence of $d_{k} \in \mathcal{D}_{k} \backslash \mathcal{D}_{i}$ such that $p_{k j}>\max \left\{W_{\alpha j}(i, j), W_{\alpha j}(N)\right\}$ and $p_{k k}>\tilde{w}_{\alpha k}(i, k)$. Consequently cartel $\{i, k\}$ does not form.

\subsubsection{Suppose that firm $i$ is the last firm to declare:}

The arguments to prove the results stated in the proposition are so close than those used in the previous case that we omit them here.

\section{Lemma 4}

Let the maximal payoff that firm $k$ can obtain in $\mathcal{V}_{\alpha}(i, k)$ subject to $P_{i}=$ $W_{\alpha i}(i, j)$ be denoted by $\tilde{w}_{\alpha k}(i, k)$.Similarly, $\tilde{w}_{\alpha k}(j, k)$ stands for the maximal payoff that firm $k$ can obtain in 2 subject to $P_{j}=W_{\alpha j}(i, j)$.

Lemma 4: Suppose all our assumptions except assumption 2 hold. Furthermore let $\mathcal{S}=\{\{i, j\},\{i, k\},\{j, k\}, N\}, \theta_{i}<\theta_{j}<\theta_{k}, \bar{q}_{j} \leq$ $\bar{q}_{i}$ and $W_{\alpha j}(i, j) \geq W_{\alpha j}(N)$. Then there exists $\bar{\alpha}<1$ such that for all $\alpha \in(\bar{\alpha}, 1) \tilde{w}_{\alpha k}(i, k)<\tilde{w}_{\alpha k}(j, k)$.

The proof is available upon request. 